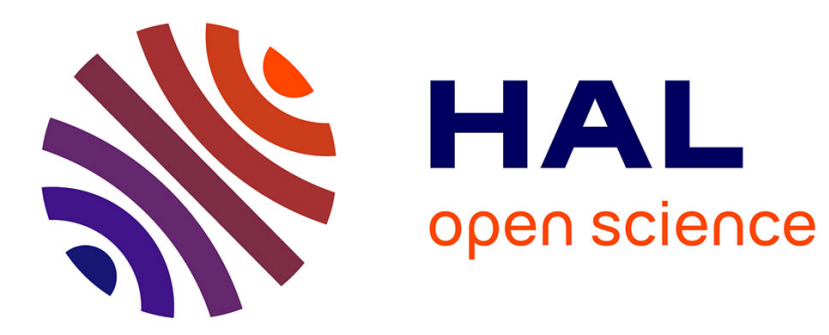

\title{
Scaling Time-Dependent Origin-Destination Matrix Using Growth Factor Model
}

Fereshteh Asgari, Ahmed Amrani, Mostepha Khouadjia

\section{To cite this version:}

Fereshteh Asgari, Ahmed Amrani, Mostepha Khouadjia. Scaling Time-Dependent Origin-Destination Matrix Using Growth Factor Model. International Conference on Transport and Smart Cities, Sep 2021, Frankfort, Germany. hal-03358508

\section{HAL Id: hal-03358508 \\ https://hal.science/hal-03358508}

Submitted on 29 Sep 2021

HAL is a multi-disciplinary open access archive for the deposit and dissemination of scientific research documents, whether they are published or not. The documents may come from teaching and research institutions in France or abroad, or from public or private research centers.
L'archive ouverte pluridisciplinaire HAL, est destinée au dépôt et à la diffusion de documents scientifiques de niveau recherche, publiés ou non, émanant des établissements d'enseignement et de recherche français ou étrangers, des laboratoires publics ou privés. 


\title{
Scaling Time-Dependent Origin-Destination Matrix Using Growth Factor Model
}

\author{
Fereshteh Asgari*, Ahmed Amrani* and Mostepha Khouadjia * \\ IRT SystemX, Saclay, France. \\ E-mail: fereshteh.asgarieirt-systemx.fr, ahmed.amranidirt-systemx.fr, \\ mostepha.khouadjiadirt-systemx. fr
}

\begin{abstract}
Demand estimation in public transport is critical for transport stakeholders. Thanks to the emerging technologies in recent years, many sources of mobility data are available to model passengers flow in public transport network. One of the most added-value mobility data is smart card OriginDestination (OD) data. These data could inform us on when, where and how flows transit within the network. The OD matrix used in this work is obtained from smart card data collected by Automated Fare Collection (AFC) system in the Greater Paris Area which is called Navigo Pass. Despite its immense value, this matrix doesn't cover the entire passenger flow. This is due to fraud, other types of tickets (e.g. the standard paper ticket) and uncertainties in destination estimates. In this paper we propose a twostep approach for correcting and scaling smart card OD matrix based on adapted Growth Factor model considering the complexity caused by temporal variation of the OD matrix. In the first step we map all the OD pairs in the OD matrix over our area of study to infer their departure and arrival stations and time. In the second step we exploit passengers' counting data and use growth factor model to scale the OD matrix to obtain a new corrected matrix which can present the real flow in the transit network. We apply our proposed methodology to scale an OD matrix constructed only from smart card validation data which presents between $40 \%$ to $65 \%$ of the overall flow. For this purpose, passengers' counting data are exploited.
\end{abstract}

\section{Introduction}

Demand estimation in public transportation network is crucial for transport operators. Thanks to the emerging technologies in recent years, many sources of data are available in the domain of mobility. Automated fare collection (AFC) system as one of these valuable data sources provides data that can be leveraged in different applications. In this work, AFC data provided by Ile-De-France Mobilités (IDFM) ${ }^{1}$ is namely called Navigo pass, which is a contact-less smart card that enables authenticated access by scanning the card at an electronic reader. The Navigo validation data contains only validation information collected at the boarding stations. Alighting station is estimated through a procedure of destination estimation. And finally the passenger flow data is aggregated in the form of OriginDestination (OD) matrix where each OD pair presents a journey considering several modes of public transportation (eg. a combination of bus and train, or train and subway, etc.). Despite its huge advantage, in many cases the OD matrix built from AFC data can partially represent the entire passenger flow. This is due to some reasons: I) a proportion of passengers use paper tickets. II) fraud and non-validation behavior can constitute a significant proportion of the flows. III) the Navigo card does not provide the destination and there are always errors in estimated destinations. IV) Last but not least, in suburban areas where stations entrance are open, non-validation percentage is high. Our preliminary analysis shows that, for our area of study depending on the location and characteristics of the station, the smart

\footnotetext{
1 IDFM is the organisation authority that controls and coordinates different transport companies operating in the greater Paris
} region. 
card data can present between $40 \%$ to $65 \%$ of the overall flow. To overcome this deficiency, we propose an approach to adjust validation OD matrix considering their spatio-temporal variation by leveraging an additional data set which is the passenger's counting data. This data set which is collected by automatic counting system contains the number of passenger's boarding on and alighting from each train at each station. This data set provided by French railway company SNCF is considered as ground truth.

To achieve our objective, growth factor model [1] from trip distribution methods is used. Trip distribution is the second step in the four step ${ }^{2}$ classic transport demand modeling [1]. Trip distribution techniques are used to obtain real OD matrix where OD matrix is partially available.

In a conventional growth factor model trip pattern is presented as an OD matrix where rows and columns represent each of the zones in the study area. In this work we are dealing with public transport stations instead of zones. The cells of each row $i$ contain the trips originating in that station which have as destinations the ones in the corresponding columns. $T_{i j}$ is the number of trips from origin $i$ to destination $j$. $O_{i}$ is the total number of trips originating in station $i$ and $D_{j}$ is the total number of trips ended at station $j$. If reliable data is available to estimate $O_{i}$ and $D_{j}$, the OD matrix can be adjusted with relative growth rates at origins and destinations. There are two constraints that should be satisfied: the sum of the trips in a row should equal the total number of trips emanating from that station and the sum of the trips in a column should correspond to the number of trips ended to that station.

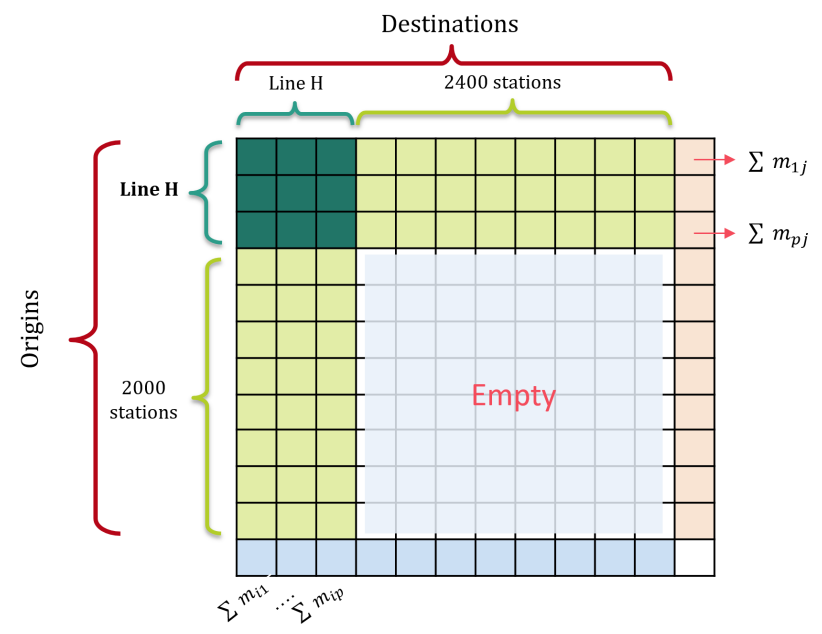

Figure 1: validation OD matrix schema

Current study is based on the principles of Growth Factor Model. However, in order to take into account spatial and temporal constraints and to make the best use of the available data, we have incorporated adaptations and improvements to the classic Growth Factor Model. The first one is that the reliable data to infer $O_{i}$ and $D_{j}$ are only available for our study area while there are many more stations where the trips are origination from/ending to. Figure 1 presents an schema of such OD matrix. As illustrated in the figure, the primary OD matrix is the smart card validation OD matrix (we will call it validation OD matrix in the rest of this paper ) which contains all the trips starting or ending in any stations of line $\mathrm{H}$, therefore all the trips are distributed in the green colored area and the subset of matrix colored in white is empty. Knowing that the ground truth is only available for the stations of line $\mathrm{H}$, we propose a mapping step to project all the trips on line $\mathrm{H}$ by estimating for each $\mathrm{OD}$ pair the boarding and alighting stations on line $\mathrm{H}$. For this intention we use a trip planner to project the trajectories on line $\mathrm{H}$ and estimate for each OD pair the station and time of boarding to /alighting from trains of line $\mathrm{H}$.

The second difference comes from temporal aspect (time dependency) of validation OD matrix which accordingly add a new dimension to the problem. The temporal resolution of validation OD matrix is 15 minutes, meaning that the smart card tap in time stamps are aggregated by 15 minutes time intervals. We know that the ticket validation time-stamp is not equal to boarding time. Moreover, the validation data does not contain the trips ending time.

2 The four steps are: 1.Trip generation 2.Trip distribution 3.Modal split and 4.Rout assignment 
With OD projection on line $\mathrm{H}$, we transform the raw validation OD matrix to an OD matrix relevant to our study area which enable us to use passenger's boarding/alighting data as reference data in OD matrix scaling. Once the new projected OD matrix is built, a temporal growth factor model will be applied to obtain the updated OD matrix. The scaled OD matrix presenting the real OD flow, will provide valuable information to transport operators. This information can be used in several applications such as demand estimation, planning [2] and the regulation [3], [4] in case of incidents.

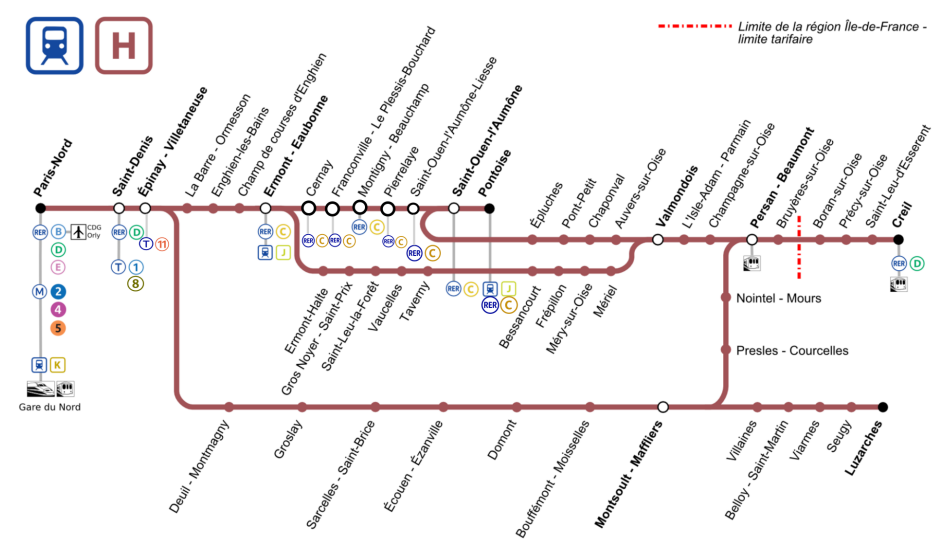

Figure 2: Line H Schema

The rest of the paper is organised as follow: In the next section we presents some of the related works and studies. Section 3 is dedicated to our proposed methodology which starts with data representation, notations and definitions. Then, we present the procedure of OD matrix scaling to obtain the updated OD matrix. Finally, we present the experiment carried out and evaluate our approach by presenting the obtained results. In section 4 we conclude with some perspectives to our works.

\section{Related Works}

In recent years many studies are performed to estimate the origin-destination matrix from smart card data. As mentioned earlier, the Origin-Destination matrix used in this work, is estimated employing Navigo Smart card data which contains the information of boarding stations. The alighting stations are estimated through a process. In general the alighting station for each passenger is estimated using trip chain model created from the sequence of validations [5], [6], [7], [8]. Along with OD matrix estimation approaches, there are studies in which supplementary source of data is used to calibrate or improve the quality of the initial OD matrix. Spurr et al. [9] have used Montreal subway smart card data to confront with household travel survey data in order to identify the bias of travel survey data and adjust the OD matrix accordingly. In another study, Savrasovs et al. [10] proposed a methodology to estimate the OD matrix based on traffic counts and confront the initial OD matrix with the information extracted from Video records. With this confrontation, they calibrate the initial OD matrix using TFlowFuzzy method. In this paper, the initial OD matrix is estimated from Smart card data in the greater Paris area and the supplementary data is the passengers counting data that will be exploited to scale the initial OD matrix in order to present the real OD flow.

\section{Growth Factor Model Based Scaling Approach}

This section covers the main contribution of our work. It starts with presenting different data sources that have been used in this work. Then OD matrix scaling method are explained in two steps: I) OD matrix Projection in which we explain how the OD matrix obtained from validation data is projected on the area of study. II) Adapted Growth factor model for temporal matrix in which we explain how to use boarding/alighting data to compute scaling factors and apply the factors to update the projected OD matrix accordingly. The section ends with presenting experiment and evaluation results. 


\subsection{Data Description}

The area of study in current work is Line $\mathrm{H}$ Transilien, a line of suburban trains which serves the north-west of greater Paris area and approximately fifty stations. The railway line carries about 250k passengers per day. There are 5 different branches in line H. Figure 2 presents a schema of line, its expansion and its correspondence with other lines. As shown in the figure, there are stations which are shared between 2 or more lines. The hub station in Paris is Paris-Nord where different subway and train lines are corresponding. Different sources of data which are used in this work are presented in continue.

(i) Smart card validation OD matrix: This OD matrix is built from the smart card validation data collected at the entrance of the stations. It is provided by IdF Mobilités following process on the smart card validation data to estimate the destinations for each Navigo validation. This OD matrix is aggregated per 15 minutes interval. That's to say, time stamp "08:00:00" means the passenger has validated his/her card between "08:00:00" and "08:15:00". Since the focus of this work is line $\mathrm{H}$, we filter all the trips starting from/ending to the stations of line $\mathrm{H}$.

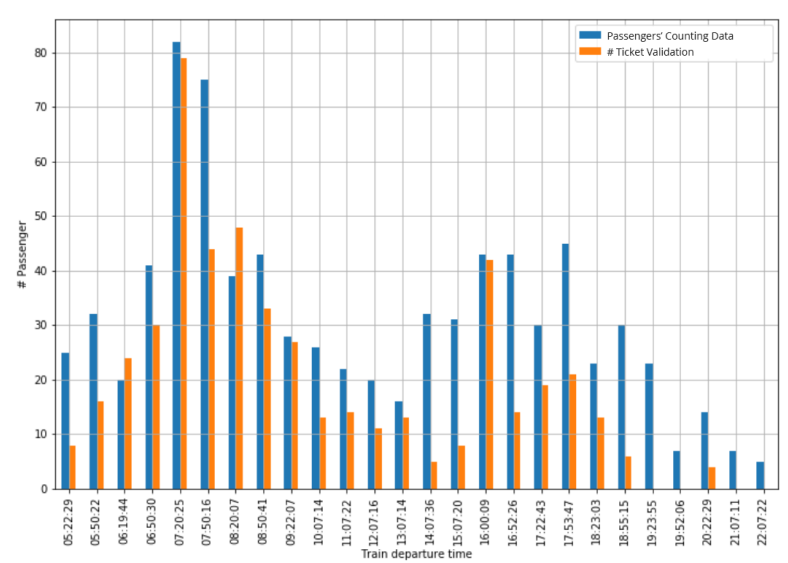

Figure 3: Box plot of number of validations compared with boarding by automatic counting data in Luzarches station

(ii) Automatic passenger's counting data is collected by sensors installed in the trains of Line $\mathrm{H}$ to count number of passengers boarding / alighting into the trains along with the train's timetable. This data set is provided by the public transport operator SNCF and used as ground truth for OD matrix scaling. Figure 3 compares number of ticket validation according to OD matrix with boarding numbers according to automatic counting data in one of the station of line $\mathrm{H}$ during one day. The difference between the two values shows the gap between validation data and real boarding which can vary in different time intervals.

(iii) Public transportation schedules presented as the General Transit Feed Specification (GTFS) is a data specification that allows public transit agencies to publish their transit data in a format that can be consumed by different software applications. GTFS is split into a static component that contains schedule, fare, and geographic transit information and a real-time component that contains arrival predictions, vehicle positions and service advisories.

\subsection{OD matrix Projection}

As mentioned earlier, each OD pair in the validation OD matrix presents a journey that can include several usage of public transport. The purpose of OD matrix projection is to estimate for each OD pair in validation OD matrix the arrival and departure time and station on the area of study which is line $\mathrm{H}$. For OD matrix projection, we use Navitia ${ }^{3}$ multimodal trip planner to provide traveler information. In order to generate the itineraries, Navitia trip planner takes two types of inputs: 1) Public transportation

3 Navitia is an open-source web API developed by Kisio Digital 
schedules in GTFS format, which was presented in data description and 2) trip information which contains the coordinates of origin and destination and the departure time. The trip planner can provide several possible itineraries to travel from origin to destination with considering different criteria in which it is important. A trip calculation component provided by the Navitia trip planner, uses a variant of the Raptor algorithm [11]. In this step for each OD pair, we take the first itinerary proposed by Navitia trip planner and extract the time stamps and stations of boarding and alighting on line $\mathrm{H}$. It is important to notice that there will be certain number of the OD pairs which are not mapped on line $\mathrm{H}$ and therefore will be removed in the projection step. It concerns mostly the case of trips from/to the stations which are shared between more than one line (station Pontoise for example). On the other hand, in the stations with lots of correspondences (Gare du Nord for example), the real number of boarding are increasing according to projected OD matrix. What is important is that in both cases, projecting the OD matrix leads to retrieve more realistic information from validation OD which can be easily compared with automatic counting data.

Table 1: Notation and Variables

\begin{tabular}{ll}
\hline Notation & \\
\hline$t$ & A time step $t \in[1, T]$ \\
station-list & stations of line $\mathrm{H}$ (list) \\
$O_{i}^{t}$ & total number of boarding at station $i$ at time $t$ according to passengers' counting data \\
$D_{j}^{t}$ & total number of alighting at station $j$ at time $t$ according to passengers' counting data \\
Matrix $m$ & input Matrix where $m_{i j}^{t}$ is the number of trips from station $i$ to $j$ at time $t$ \\
Matrix $M$ & Result Matrix where $M_{i j}^{t}$ is the number of trips from station $i$ to $j$ at time $t$ \\
$A_{i}^{t}$ & Scaling factor for line $i$ at time $t$ \\
$B_{j}^{t}$ & Scaling factor for column $j$ at time $t$ \\
\hline
\end{tabular}

\subsection{Growth Factor model for temporal OD matrix}

Growth factor model is a method which respond to relative growth rates at origins and destinations. In this part, we present the adapted growth factor model in which temporal dimension is taken into consideration. Lets present the projected validation OD matrix as matrix $m$ where $m_{i j}^{t}$ is the number of trips from origin $i$ to destination $j$ departed at time interval $t$. The objective is to find matrix $M$ where $M_{i j}^{t}$ is the overall number of trips from origin $i$ to destination $j$ at time interval $t$. Capital letters represent the objective matrix or the values we are trying to model for the corresponding modelling period. Passengers' counting data is used to estimate $O_{i}^{t}$ and $D_{j}^{t}$. $O_{i}^{t}$ is the number of trips originating from station $i$ at time interval $t$ and $D_{j}^{t}$ is the number of trips ending in destination $j$ at time interval $t$. (table 1 presents these notations).

In a doubly constraint Growth Factor model, there are two constraints which should be respected, constraint 1: the sum of all the trips in a row should correspond to the number of trips starting from that origin:

$$
\sum_{j} M_{i j}^{t}=O_{i}^{t}
$$

constraint 2: the sum of all the trips in a column should correspond to the number of trips ending in that station:

$$
\sum_{i} M_{i j}^{t}=D_{j}^{t}
$$

The main adaptation in this model is incorporating time dimension in matrix scaling. The objective matrix $M_{i j}^{t}$ is calculated in an iterative process. It is important to notice that when we split a static OD matrix to a sequence of temporal matrix, these sequences are dependent to each other and therefore, the process of adjustment needs to be performed for the whole sequence of matrix. We start with computing 
the scaling/adjustment factor for each origin station $i$ and for each time interval $t$ :

$$
\forall t, i \quad A_{i}^{t}=\frac{O_{i}^{t}}{\sum_{j} m_{i}^{t}}
$$

Once the factor table is computed, it is used directly to adjust the matrix $m$ :

$$
\forall t, i \quad M_{i j}^{\prime} t=A_{i}^{t} \times m_{i j}^{t}
$$

where matrix $M^{\prime}$ is the scaled OD matrix using only information of boarding data at the origin stations. In the same way we compute factor $B_{j}^{t}$ :

$$
\forall t, j \quad B_{j}^{t}=\frac{D_{j}^{t}}{\sum_{i} M_{j}^{\prime t}}
$$

and consequently matrix $M$ is computed as:

$$
\forall t, j \quad M_{i j}^{t}=B_{j}^{t} \times M_{i j}^{\prime t}
$$

Matrix $M_{i j}^{t}$ is the scaled OD matrix which presents the number of trips from station $i$ to station $j$ at time interval $t$. Algorithm 1 below summarizes one iteration of the matrix scaling procedure using Growth Factor Model as explained in this section. This procedure can continue for several iterations until reaching the optimal criteria. The iteration can stop when the overall difference between the real flow (according to the passenger counting data) and the scaled matrix is less than a defined threshold. (eg. a threshold of 5\%).

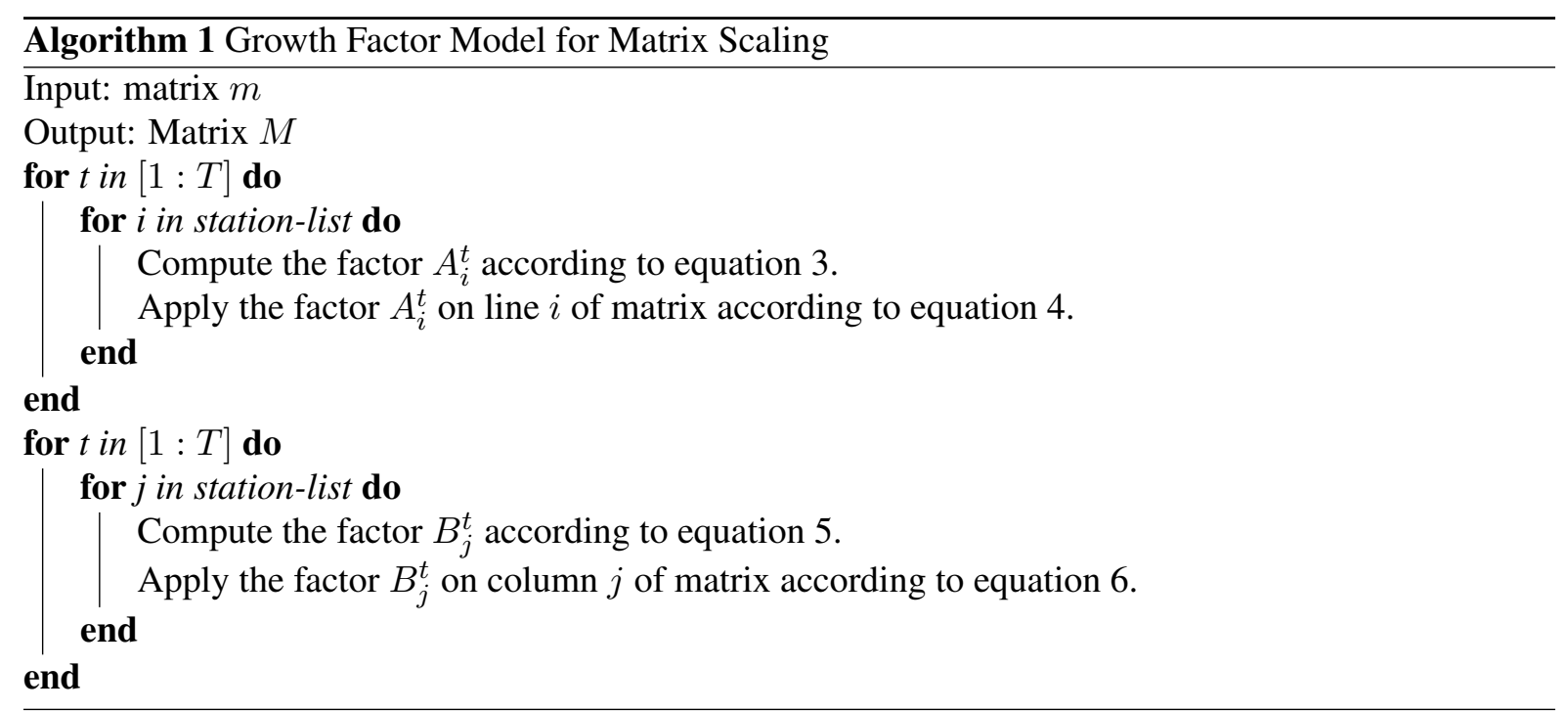

In this section we presented the procedure of scaling the validation OD matrix $m$ by exploiting the passengers' counting data (as $O_{i}$ and $D_{j}$ ) to obtain the OD matrix which is close to the real OD flow. The scaled OD matrix can lead to a better estimation of demand in public transport.

\subsection{Experiment}

The proposed temporal growth factor model is applied on real OD matrix corresponding to a period of 4 months from September to December 2017. The ground truth which are the passengers' counting data covers also the same period. As mentioned earlier, the area of study corresponds to the stations of the line H. The OD matrix scaling starts with extracting daily validation OD matrix. This matrix corresponds to all the trips starting or ending at stations of line H. Initially, the validation OD matrix is aggregated in the intervals of 15 minutes. Therefore, we start with OD matrix dis-aggregation. As explained in 
section 3.2. the OD matrix scaling starts with OD projection. For this purpose, we use Navitia trip planner. For each OD pair, the first trajectory proposed by the trip planner is considered to estimate the departure/arrival time and station on line $\mathrm{H}$. In this step, the total number of OD pairs projected on the line $\mathrm{H}$ represents approximately $70 \%$ of the total number of OD pairs. This shows the important role that the projection phase plays in filtering the initial matrix. This can be explained with the fact that in the stations of line $\mathrm{H}$ which are shared between other train lines (eg. station Pontoise shared between line $\mathrm{H}, \mathrm{C}$ and $\mathrm{J}$ ), some of the ticket validations correspond to the trips in which the passengers take other lines. As consequence, after projecting the validation OD matrix on line $\mathrm{H}$, these trips are removed.

To estimate $O_{i}$ and $D_{j}$ as reference values, we use data of 3 months and test the scaling on another day that was not used for estimation. We select a set of days as reference days to estimate the value of boarding/alighting as reference values $\left(O_{i}\right.$ and $\left.D_{j}\right)$. In selecting the reference days scholar vacations are excluded. We also removed the abnormal days where the gap between daily number of boarding and the average daily value, are higher than 5\%. For each day of week, reference days are selected from the same day type (eg. the data of 6 Thursdays used to estimate the reference value for another Thursday). Confirming the seasonal and weekly pattern of passenger's counting data, some variation is still exist in these data. Fig. 4 illustrates this variation by value of standard deviation (pink area) for two stations of the line H. Standard deviation variation can be interpreted as an indicator of the degree of reliability for reference value estimation. Considering these variation which could affect the final reference value, the median value of boarding /alighting data among all the selected days from automatic counting data is estimated as $O_{i}^{t}$ and $D_{j}^{t}$. The daily projected OD matrix is used as matrix $m$. We use three different time intervals $t=15,30$ and 60 minutes to apply matrix scaling. The result of OD matrix scaling experiments are presented in the next section.
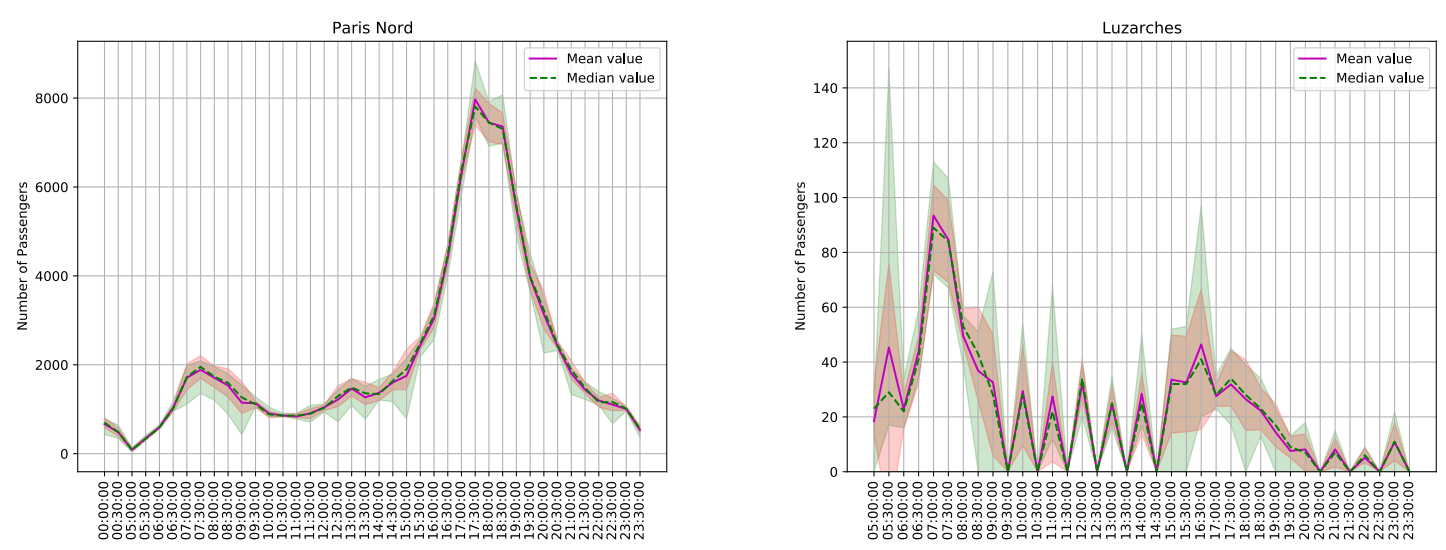

Figure 4: Passenger's boarding from automatic counting systems for two stations of Paris Nord and Luzarches. Data comes from Thursday in September, October and November 2017. Holidays and abnormal days are excluded. Pink area shows the standard deviation around the median of the counting values.

\subsection{Results Evaluation}

Six stations among all the stations of line $\mathrm{H}$ were chosen to demonstrate the results of each step. These stations are 'Gare du Nord', 'Montigny-Beauchamp', 'Ermont-Eaubonne', 'Pontoise', 'Luzarches' and 'Sarcelles st Brice'. The station 'Gare du Nord' is a hub station in Paris. The stations 'MontignyBeauchamp', 'Ermont-Eaubonne' and 'Pontois' are shared stations between several lines. Two stations 'Luzarches' and 'Sarcelles st Brice' belong to a simple branch of the line $\mathrm{H}$ where 'Luzarches' is in the beginning of the line and 'Sarcelles st Brice' is in the middle. Fig. 5 presents, for a single test day, a comparison between the number of boarding extracted from the final scaled matrix (scaled projected OD), the number of boarding obtained from the projected matrix (Projected OD), the number of validations (Validation OD) and the number of boarding obtained from counting data (Ground truth). 


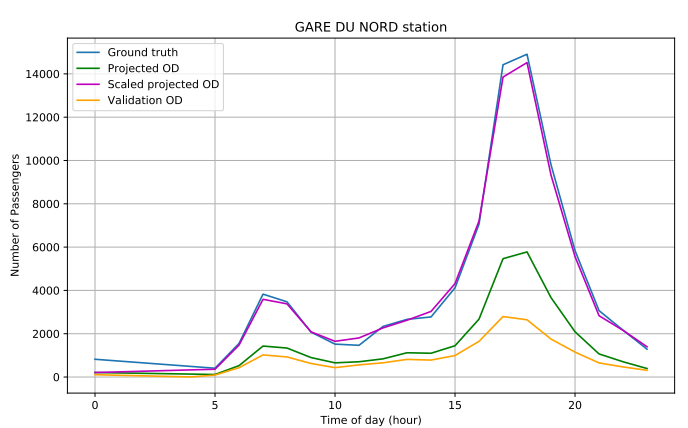

(a) Station Paris Nord

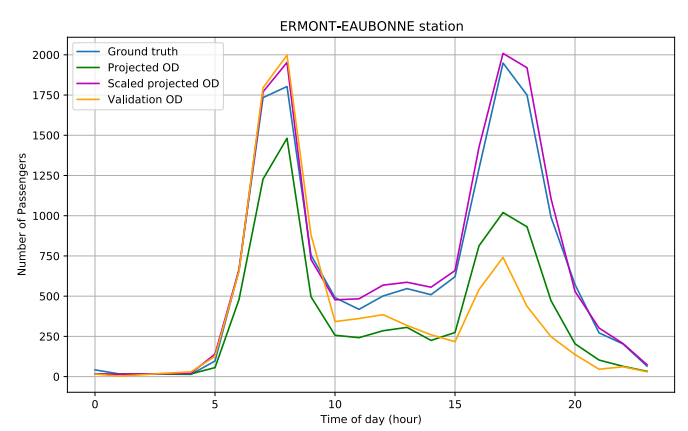

(c) Station Ermont-Eaubonne

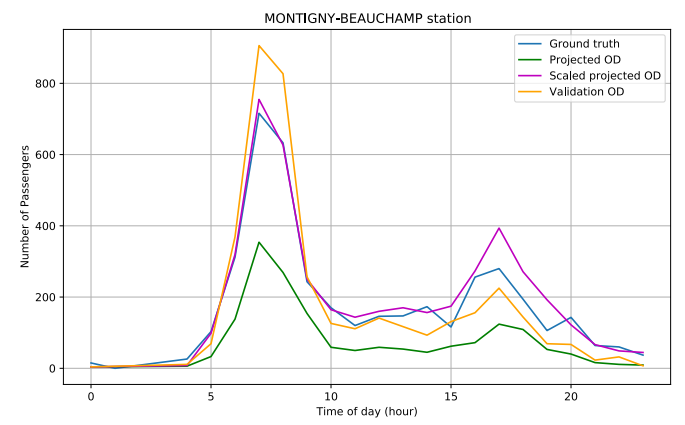

(e) Station Montigny-Beauchamp

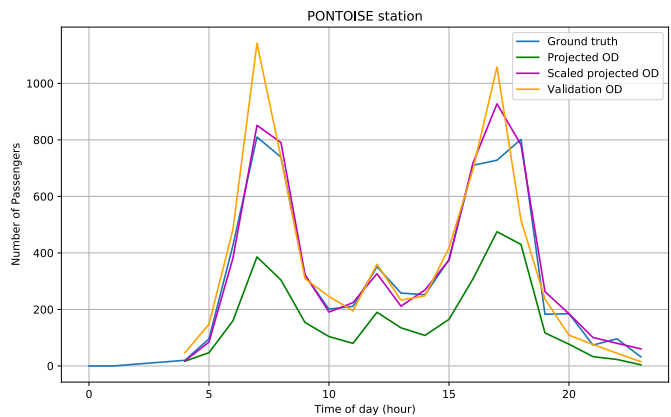

(b) Station Pontoise

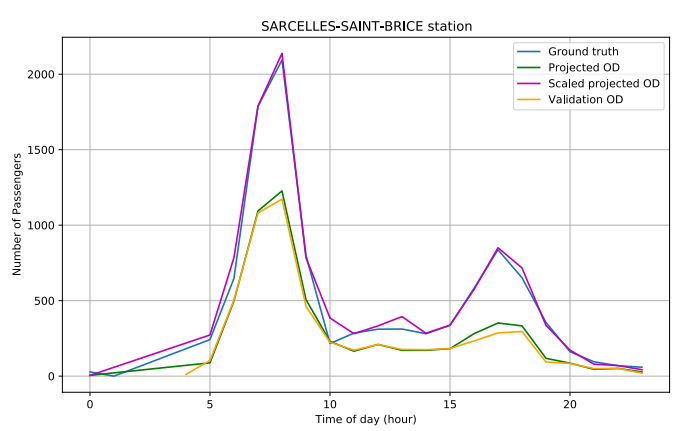

(d) Station Sarcelles Saint-Brice

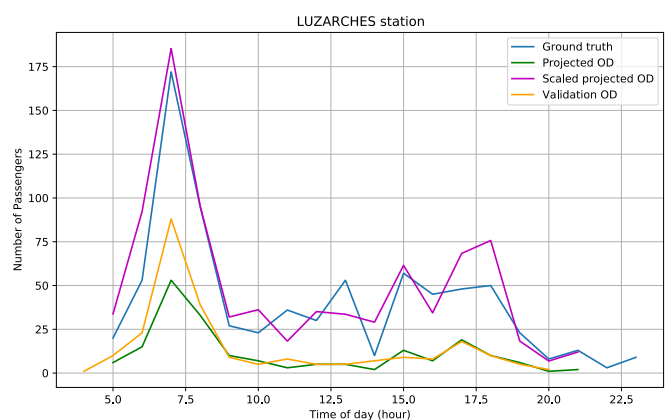

(f) Station Luzarches

Figure 5: comparing the number of ticket validation (orange) with ground truth (blue), projected boarding (green) and scaling result (magenta) for one day

We can observe in these results different behaviours of stations by comparing ticket validation number and projected boarding number. In station 'Gare du nord' in Fig. 5a as a hub and transfer station, the number of boarding after projection is almost twice as the number of validations. On the other hands, stations such as 'Pontoise' Fig. 5b and 'Montigny Beachamp' Fig. 5e which both are served by several train lines, shows inverse behaviours and number of boarding after the projection are less than validation number per time intervals. Station Ermont-Eaubonne demonstrates two different behaviours in the morning and evening peak hours. Stations such as 'Luzarches' Fig. 5f and Fig. 5d 'Sarcelles Saint Brice' are simple station with not much difference between number of validations and projected boarding. The considerable differences between validation numbers and projected boarding, shows the importance of projection step in our OD matrix scaling methodology.

To evaluate the performance of obtained results, we computed Weighted Average Percentage Error (WAPE) by comparing ground truth from real counting data and scaled OD matrix. Table 2 presents 
Table 2: Weighted Absolute Percentage Error (WAPE) for selected stations average on 3 days of different types

\begin{tabular}{l|c|c|c} 
& \multicolumn{3}{|c}{ Selected Time Intervals } \\
\hline Stations & $15 \mathrm{~min}$ & $30 \mathrm{~min}$ & $60 \mathrm{~min}$ \\
\hline \hline Gare du Nord & $16.2 \%$ & $13.3 \%$ & $9.8 \%$ \\
\hline Pontoise & $23.7 \%$ & $15.5 \%$ & $14.5 \%$ \\
\hline Sarcelles St Brice & $23 \%$ & $15 \%$ & $9.1 \%$ \\
\hline Montigny-Beauchamp & $26 \%$ & $22 \%$ & $15 \%$ \\
\hline Ermont-Eaubonne & $19.7 \%$ & $16.5 \%$ & $11 \%$ \\
\hline Luzrches & $44.5 \%$ & $43.2 \%$ & $36.7 \%$ \\
\hline
\end{tabular}

the WAPEs for scaling method applied on three different time discretization intervals for the selected stations. Fig. 6 also illustrates for each station the computed WAPEs in $\mathrm{x}$ axis and the average number of passengers in y axis. As table shows, the scaling method for 60 minutes interval leads to a better estimation. In addition, we can see from Fig. 6 that the higher error values correspond to the stations with few number of passengers and our investigation confirms that these small stations in suburban area have open entrance. As a result, at these stations very few or zero ticket validation is recorded. Knowing that growth factor model is sensitive to zero, our approach fails to retrieve the overall trips in these stations. However these six stations altogether serve less than $1 \%$ of daily passengers and in the stations with higher ridership the proposed approach leads to more reliable scaling results. In general we succeed to retrieve up to $95 \%$ of daily trips thanks to OD matrix scaling method.

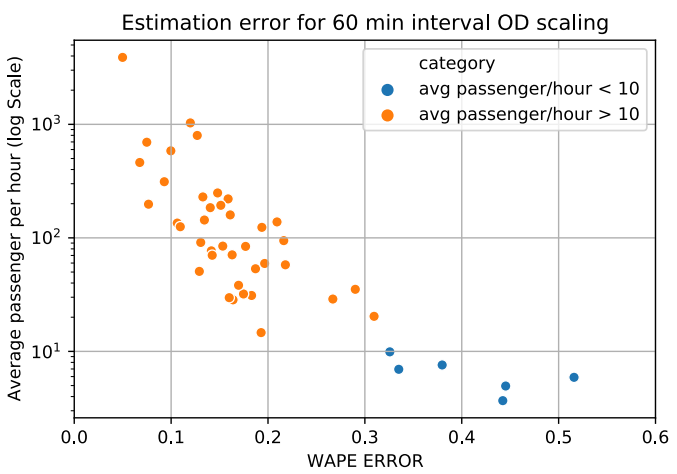

Figure 6: WAPE estimation error for all the stations of line $\mathrm{H}$ with average number of passengers at each station per time interval. The six stations colored in blue serve less than $1 \%$ of daily passengers according to counting data.

\section{Conclusion and Perspectives}

In this paper we have proposed a time-dependent OD matrix scaling method based on growth factor model. The proposed approach aims to scale-up the smart card based OD passenger flows in order to be more representative and close to real transit flows. For that purpose, we used passengers boarding and alighting data as a ground truth. The proposed matrix scaling method can be used as an inexpensive solution to scale up validation OD matrix which can lead to better estimation of demand in public transport stations. To improve the result of OD matrix scaling method, we would like to enhance the robustest of the model in dealing with missing data. Using other methods which are resilience to zero such as Gravity model can be taken into the consideration as well. In addition, making use of other sources of data such as survey data could help to improve the reliability of reference data. For the future works, we aim to use the scaled OD matrix as a starting point for assignment process to estimate 
important network indicators such as load on board, number of the passengers waiting on the platform or at station, travel times, etc. These indicators can help operators to better plan and regulate their transportation system.

\section{Acknowledgements}

The authors wish to thank the transport organization authority Île-de-France Mobilités (IDFM), and SNCF operator for providing the data, and Kisio Digital for their support in making use of their journey planner Navitia (www.navitia.io). This research work is a part of IVA Project which aims to enhance traveler information and carried out under the leadership of the Technological Research Institute SystemX, with the support of public funds given into the scope of the French Program "Investissements d'Avenir".

\section{References}

[1] Juan de Dios Ortuzar and Luis G.Willumsen. Modeling Transport. John Wiley Sons,Ltd, 2011.

[2] Marc Naumann, Leena Suhl, and Stefan Kramkowski. A stochastic programming approach for robust vehicle scheduling in public bus transport. Procedia Social and Behavioural Science., 20:826-835, 2011.

[3] G.E. Sánchez-Martínez, H.N. Koutsopoulos, and N.H.M. Wilson. Real-time holding control for high-frequency transit with dynamics. Transportation Research Part B: Methodological, 83:1-19, 2016.

[4] Doris Sáez, Cristián E. Cortés, Freddy Milla, Alfredo Núñez, Alejandro Tirachini, and Marcela Riquelme. Hybrid predictive control strategy for a public transport system with uncertain demand. Transportmetrica, 8(1):61-86, 2012.

[5] Martin Trépanier, Robert Chapleau, and Nicolas Tranchant. Individual trip destination estimation in a transit smart card automated fare collection system. Journal of Intelligent Transportation Systems Technology Planning and Operations, 11, 2007.

[6] Di Huang and Jun Yu. A method for bus od matrix estimation using multisource data. Journal of Advanced Transportation, Article ID 5740521, 2020.

[7] Azalden A. Alsger, Mahmoud Mesbah, Luis Ferreira, and Hamid Safi. Use of smart card fare data to estimate public transport origin-destination matrix. Journal of Transportation Research Board, pages 88-96, 2019.

[8] Mona Mosallanejad, Sekhar Somenahalli, and David Mills. Origin-destination estimation of bus users by smart card data. Computational Urban Planning and Management for Smart Cities. CUPUM, 2019.

[9] Tim Spurr, Robert Chapleau, and Daniel Piché. Use of subway smart card transactions for the discovery and partial correction of travel survey bias. Journal of the Transportation Research Board, No. 2405:57-67, 2014.

[10] Mihails Savrasovs and Irina Pticina. Methodology of od matrix estimation based on video recordings and traffic counts. Procedia Engineering, 178:289-297, 2017.

[11] Daniel Delling, Thomas Pajor, and Renato F. Werneck. Round-based public transit routing. Proceedings of the Meeting on Algorithm Engineering and Experiments (ALENEX), page 826-835, 2012. 